Ann. Biol. anim. Bioch. Biophys., I962, 2 (4), 345-360.

\title{
RÉPARTITION DES FIBRES NERVEUSES SENSITIVES ET MOTRICES DANS LE TRONG VAGAL THORACIQUE DU MOUTON
}

\author{
M. DUSSARDIER ( ${ }^{(1)}$ \\ avec la collaboration technique de Colette Lavenet et J.-L. Dured \\ Station de Recherches de Physiologie animale, Centre national do Recherches zootechniques, \\ Jouy-en-Josas (Seine-et-Oise)
}

\section{SOMMAIRE}

Le ganglion plexiforme, le ganglion jugulaire et les branches intrathoraciques du vague ont été étudiés chez le Mouton.

La technique de dégénérescence rétrograde a permis de montrer que les corps cellulaires des fibres sensitives vagales destinées à l'abdomen se trouvent dans le ganglion plexiforme, et non dans le ganglion jugulaire. Contrairement à ce qui se passe chez la plupart des autres Mammifères, chez les Ruminants les branches vagales destinées à l'abdomen contiennent un grand nombre de fibres myélinisées ( 32000 environ au niveau du diaphragme). On peut estimer que 83 p. Ioo d'entre elles ont un diamètre inférieur à 3,5 microns. Les plus grosses (I6 p. Io0) ont de 3,5 à 6,5 microns. Les fibres amyéliniques n'ont pas été comptées.

Des nerfs normaux ont été comparés avec des nerfs ayant subi une dégénérescence wallérienne à la suite d'une vagotomie effectuée soit au-dessus du ganglion plexiforme, soit en région moyenne de l'encolure. On peut admettre, sur la base de ces expériences, qu'un nerf normal contient $68 \mathrm{p}$. I00 de fibres efférentes d'origine bulbaire et $30 \mathrm{p}$. Ioo de fibres sensitives issues du gan. glion plexiforme. Deux pour cent environ des fibres thoraciques ne dégénèrent pas après une vagotomie cervicale, et ont donc une autre origine; leur signification est discutée.

Les fibres sensitives et les fibres motrices ont un spectre de diamètre très voisin. Il n'est donc certainement pas possible de réaliser une destruction ou un blocage physiologique des unes sans léser ou bloquer les autres, ce qui aurait pourtant été précieux dans de nombreuses expériences.

\section{IN'TRODUCTION}

Afin de préciser l'importance exacte du rôle joué par les intérocepteurs dans la physiologie des viscères, il serait utile de pouvoir sectionner les fibres sensitives sans léser les fibres motrices. Au niveau du pneumogastrique, malheureusement, cette expérience est irréalisable, car les deux types de fibres sont intimement mêlées,

(1) Adresse actuelle : Laboratoire de Physiologie générale, Faculté des Sciences, Place Victor-Hugo, Marseille $\left(3^{\mathrm{e}}\right)$. 
même au niveau des racines; la distinction qui est faite parfois entre un groupe de racines dorsales sensitives et un groupe de racines ventrales motrices semble n'avoir en effet qu'une valeur approximative (FOLEY et DU BoIS, I934; BONVALLET et SIGG, I958). La séparation serait peut-être meilleure au niveau du ganglion plexiforme, car divers auteurs ont montré que les fibres motrices ne le traversent pas, mais se groupent en faisceaux qui sont accolés à sa surface (FOLEY et DU BOIS, I934; RANSON et al., I933; HoFFMAN et KUNTZ, I957). Il s'agit cependant d'une simple séparation histologique : les deux troncs nerveux ne sont pas séparables par la dissection, sauf chez le Porc (LESBre et MAIGNON, Igo8 ; Dussardier, inédit). La séparation histologique elle-même n'est d'ailleurs pas absolue, car RANson et al. (I933) ont vu un certain nombre de fibres motrices traverser le ganglion plexiforme en se mêlant aux neurones.

A défaut d'une séparation chirurgicale des fibres sensitives et motrices, on aurait pu envisager de réaliser un blocage physiologique différentiel des deux types de fibres à l'aide de l'anesthésie, du froid (Douglas et MALCor.M, r955) ou du courant électrique (BISHOP et HeINBECkER, I935; LAPORTE et BESSOU, I959). Il faudrait pour cela que le spectre de diamètre des deux types de fibres soit différent. N'ayant pas trouvé dans la bibliographie des données suffisamment précises sur ce point, nous avons été amené, dans le cadre d'une étude consacrée au contrôle nerveux de la motricité gastrique des Ruminants (Dussardier, I960), à effectuer une étude histologique du vague. L'exposé de ces recherches, effectuées à l'aide des techniques de dégénérescence wallérienne ou rétrograde, et essentiellement orientées vers la connaissance des branches vagales à destination abdominale, fait l'objet du présent mémoire.

\section{TECHNIQUES}

Certaines des pièces anatomiques ont été prélevées sur des animaux ayant subi au préalable une vagotomie aseptique. La technique utilisée pour effectuer la vagotomie intrathoracique a déjà été décrite (DUSSARDIER, I961). La vagotomie cervicale, faite en région moyenne du cou, ne pose aucun problème.

Pour réaliser une section de nerf pneumogastrique entre le crâne et le ganglion plexiforme, nous avons utilisé une technique plus simple que celle proposée par APPLETON et WAITES (1957) pour la découverte du ganglion sympathique cervical supérieur du Mouton. L'incision cutanée est faite juste en avant de l'aile de l'atlas, et parallèlement à elle. Elle monte assez haut vers la base de l'oreille mais n'a que cinq centimètres de long. On pénètre dans la profondeur en décollant vers l'arrière la glande parotide, ce qui permet de la repousser vers l'avant. On découvre alors facilement l'apophyse styloïde recouverte par les muscles qui s'insèrent sur elle. Le ganglion plexiforme se trouvant sous cette apophyse, celle-ci doit être enlevée. Les muscles qui la recouvrent et le périoste étant incisés, l'os est décortiqué, et l'on réalise une section sous-périostée à l'aide d'une pince gouge (la résection doit être sous-périostée pour éviter de léser une artère qui passe sous l'apophyse stylö̈de). On découvre alors facilement le ganglion plexiforme : il ne se traduit pas par un élargissement du nerf, mais il se trouve au niveau du point d'émergence du nerf laryngé externe. Le point le plus délicat de l'opération réside dans le fait que le grand hypoglosse, le pneumogastrique et le spinal sont à ce niveau étroitement accolés. Il est difficile de réaliser une dissection qui ne lèse pas l'un des trois nerfs.

Nous n'avons utilisé que des techniques histologiques courantes : coloration des cellules ganglionnaires à la thionine, des fibres myélinisées par immersion pendant huit jours dans une solution d'acide osmique à I p. Ioo.

Pour effectuer le dépouillement quantitatif de nos données histologiques, les coupes ont été projetées sur un papier-calque, et nous avons dessiné le contour des fibres. Puis, afin d'avoir plus de précision, les dessins ont été projetés à leur tour à l'aide d'un agrandisseur photographique. Nous avons alors évalué le diamètre des différentes fibres en comparant leur image à une série de modèles. Le système de projection utilisé a été mis au point par J. VERGE (Ingénieur à l'I. N. R. A.). 


\section{RAPPELS ANATOMIQUES}

L'anatomie du nerf pneumogastrique des Ruminants a fait récemment l'objet de deux études approfondies (HABEL, I956 ; DoughERTY, I958). Nous voudrions donc insister simplement sur deux points de nomenclature.

Après avoir émis le nerf laryngé inférieur (nerf récurrent) et des rameaux cardiaques, chacun des nerfs vagues se divise, en arrière du cœur, en une branche supérieure et une branche inférieure (fig. I). Les deux branches inférieures (gauche et droite) se réunissent très précocement, donnent naissance à cet endroit atux nerfs bronchiques, et se continuent jusque dans l'abdomen sous forme d'un tronc unique qui suit la face inférieure de l'œsophage à une certaine distance. Les deux branches

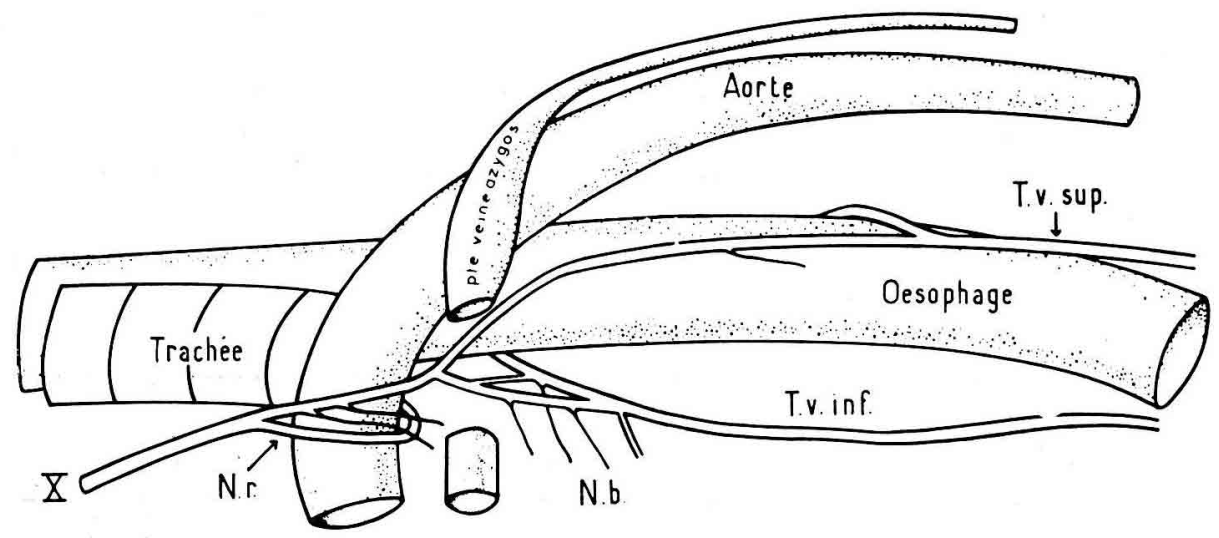

FIG. I. - Schéma du vague thoracique

$\mathrm{X}=$ nerf pneumogastrique, N. r. = nerf récurrent (laryngé inférieur), N. b. = nerf bronchique, $\Gamma$. $\mathrm{v}$. sup. = tronc vagal thoracique supérieur, $\mathrm{T}$. $\mathrm{v}$. inf. $=$ tronc vagal thoracique inférieur. La petite veine azygos a été sectionnée.

Il existe des variations importantes d'un animal à l'autre, en particulier pour le lieu d'émergence des nerfs bronchiques.

supérieures, situées à la surface de l'œsophage, se réunissent beaucoup plus tardivement que les branches inférieures ; le tronc unique, qui suit de près la face supérieure de l'œsophage, se rend également dans l'abdomen.

L,es anatomistes français (MONTANE et BOURDELLE, I9I7 ; LESBRE, I923) désignent les nerfs dont nous venons de parler sous le nom de "cordon ou nerf cesophagien supérieur " et de " cordon ou nerf œesophagien inférieur ". Ce qualificatif d' " œesophagien " basé seulement sur des rapports de voisinage ne nous paraît pas correct ; il pourrait faire croire que les nerfs se terminent dans l'œsophage. Nous préférons parler du tronc vagal supérieur et du tronc vagal inférieur, constitués respectivement par la fusion des deux nerfs vagues supérieurs (gauche et droit) et des deux nerfs vagues inférieurs (gauche et droit).

Il est essentiel de noter que les dénominations souvent utilisées, de vague gauche pour le tronc thoracique supérieur, et de vague droit pour le tronc thoracique inférieur 
ne sauraient en aucun cas être retenues, car elles ne correspondent à rien. Les fibres nerveuses provenant de gauche ou de droite se retrouvent en nombre sensiblement égal dans le tronc dorsal comme dans le tronc ventral.

\section{RÉSULTATS ET DISCUSSION}

\section{I. - LES GANGLIONS SENSITIFS}

\section{Résultats}

Che $\%$ les petits Ruminants (Mouton, Chèvre) la présence du ganglion plexiforme ne se manifeste pas par un élargissement du tronc vagal, comme cela se produit par exemple chez le Porc. On trouve cependant facilement le ganglion, car il est situé de part et d'autre du point d'émergence du nerf laryngé externe. Sur des coupes longitudinales du vague, il apparaît comme un amas de cellules long d'au moins Io $\mathrm{mm}$. L es cellules sont serrées dans la région médiane, et plus lâches aux extrémités ; elles sont parfois suffisamment clairsemées vers les extrémités pour donner l'impression d'être distribuées en plusieurs amas. Les cellules dont le diamètre varie de 30 à $70 \mu$ apparaissent sphériques ou ovoïdes. Le noyau est souvent nettement excentré; il y a souvent deux nucléoles. La substance tigroïde se colore très fortement par la thionine. trique.

Le ganglion jugulaire se trouve sur le trajet intra-osseux du nerf pneumogas-

Si l'on sectionne le tronc vagal supérieur (intrathoracique) chez un jeune animal, et que l'on prélève les ganglions au bout d'une dizaine de jours, on constate la présence dans le ganglion plexiforme d'un nombre considérable de cellules qui présentent les signes typiques de la dégénérescence rétrograde : chromatolyse, déplacement latéral du noyau qui déforme le bord de la cellule et qui peut même en être expulsé (fig. 2). Nous n'avons pas eu l'impression que la dégénérescence intéresse une région particulière du ganglion plexiforme, ni qu'elle touche d'une manière préférentielle des cellules plus ou moins grosses. Nous n'avons cependant pas effectué une étude approfondie de ce problème. A la suite de la section nerveuse considérée il n'existe pas de cellules dégénérées dans le ganglion jugulaire.

Si l'on sectionne le vague entre le bulbe et le ganglion plexiforme, les cellules de ce ganglion ne semblent pas subir de dégénérescence rétrograde.

\section{Discussion}

Nous ne connaissons pas de travail consacré au ganglion plexiforme des Ruminants, mais nos observations concordent avec celles des auteurs qui ont étudié les ganglions vagaux des Monogastriques (Molmant, I9I3; Mohiuddin, I953). Elles conduisent à admettre que, conformément à ce qui est couramment admis, le ganglion plexiforme contient des cellules pour le vague abdominal, tandis que le ganglion jugulaire n'en contient pas (selon DU Bors et FOLEY I937, au moins 75 p. IOo des fibres fournies par ce dernier ganglion sont destinées aux nerfs auriculaires). 


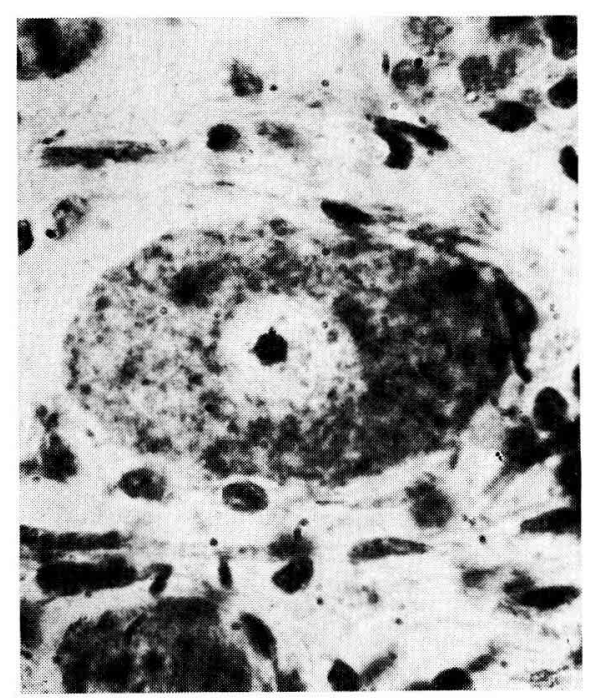

A

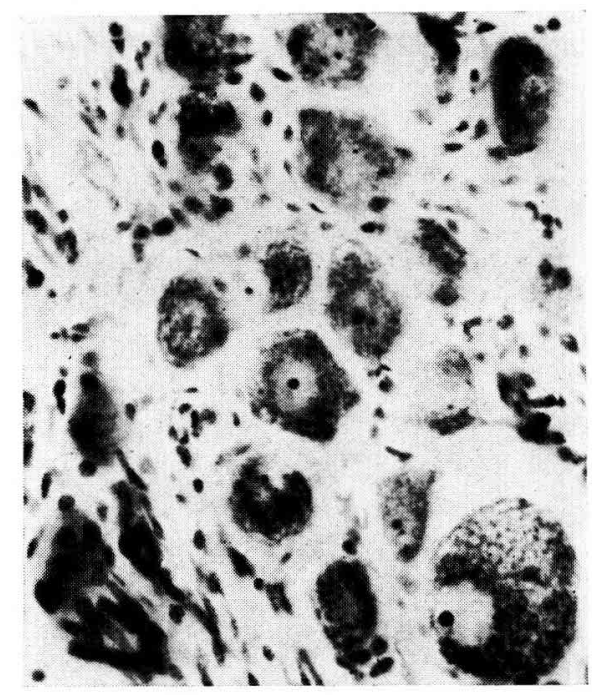

C

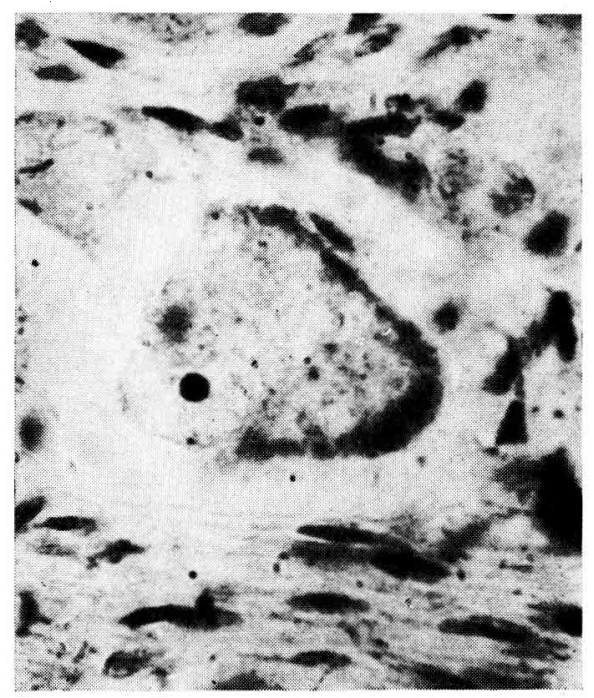

B

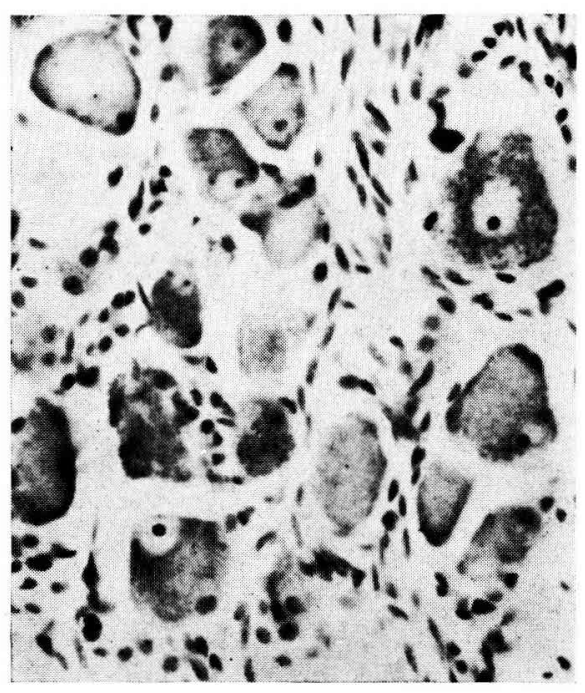

D)

FIG. 2. - Dégénérescence rétrograde des neurones du ganglion plexiforme à la suite d'une section du nerf vague thoracique supérieur (NISSL).

A. - Cellule normale.

B. - Cellule dégénérée. Noter que le noyau fait saillie hors de la cellule.

C. - Ganglion droit normal.

D. - Ganglion plexiforme gauche dégénéré.

Grossissement : I ooo fois pour $A$ et $B, 375$ fois pour C et $D$. 
Le fait que les cellules du ganglion plexiforme ne manifestent pas de signes de dégénérescence lorsque l'on sectionne la branche axonique de leur prolongement entre le bulbe et le ganglion, est en accord avec de nombreuses observations faites dans diverses espèces au niveau du ganglion plexiforme (ANDERSON, I902; KOREICHA et RAPPOPORT, I929; SATO, I933) ou des ganglions spinaux (LuGARO, I\$96 ; HINSEY et al., I937 ; KIMMEL et MOYer, I947). Seul Nikiforov (I957) est d'un avis contraire.

\section{2. - LE VAGUE THORACIQUE NORMAL}

Le vague cervical est très hétérogène et contient un grand nombre de fibres sans intérêt pour la physiologie gastrique. Nous avons donc limité notre étude à la portion du vague thoracique située en arrière de l'émergence des nerfs récurrents.

\section{Résultats}

Les deux nerfs vagues thoraciques supérieurs se rejoignant tardivement pour constituer le tronc vagal supérieur, il est facile de les étudier séparément. Ils mesurent environ de I à I,2 millimètres de diamètre et sont constitués de plusieurs faisceaux de taille très inégale; selon les animaux nous avons trouvé de 8 à 35 faisceaux. En arrière du lieu d'émergence des nerfs bronchiques, les deux nerfs thoraciques inférieurs sont déjà réunis en un tronc unique qui mesure de $I$ à $I, 5$ millimètres de diamètre et qui peut contenir de 6 à I 8 faisceaux de fibres. Les faisceaux sont séparés par des gaines conjonctives importantes.

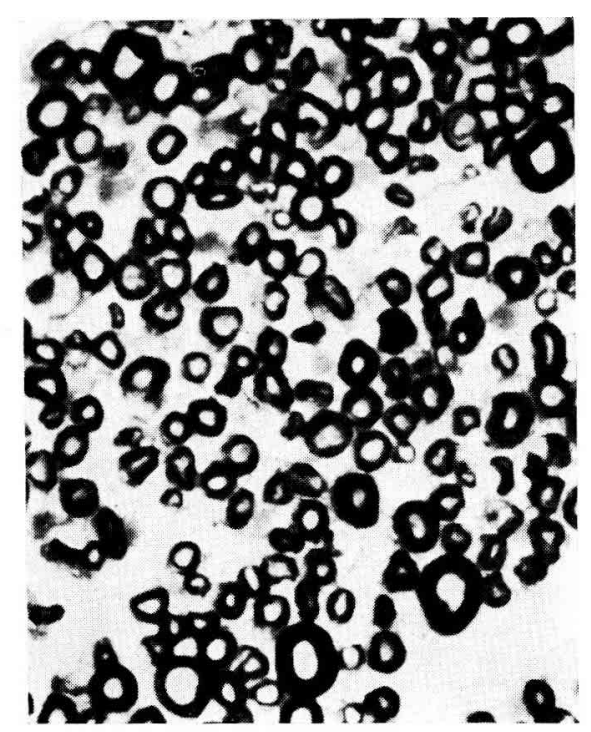

$A$

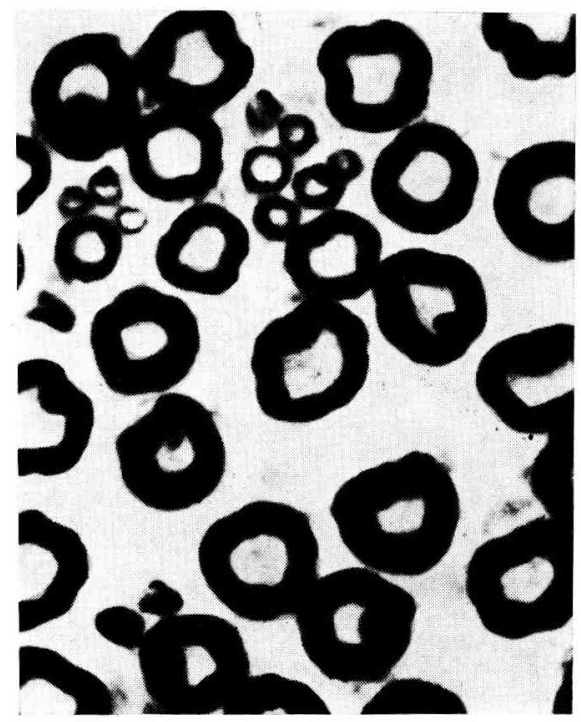

$\mathrm{B}$

FIG. 3. - Neris colorés par lacide osmique

A. - Nerf vague thoracique supérieur.

B. - Nerf phrénique.

Grossissement I ooo fois. 
Le nombre des fibres myélinisées contenues dans ces nerfs est considérable: Io 000 à I2 ooo dans chacun des nerfs supérieurs, 8 o0o à Io ooo dans le tronc com. mun inférieur. Nous pouvons donc estimer à environ 32 o0o le nombre des fibres vagales myélinisées qui traversent le diaphragme. Nous n'avons pas cherché à évaluer le nombre des fibres amyéliniques, mais il ne doit pas être considérable par rapport au nombre des fibres myélinisées car celles-ci sont relativement serrées (fig. 3).

La figure $4 \mathrm{~A}$ représente le spectre de diamètre des fibres myélinisées d'un nerf thoracique supérieur, établi d'après la mesure de 7600 fibres d'un même nerf. Dans le cas considéré $83 \mathrm{p}$. Ioo des fibres ont un diamètre inférieur à $3,5 \mu ;$ I $6 \mathrm{p}$. Ioo ont de 3,5 à $6,5 \mu ;$ I p. Ioo a plus de $6,5 \mu$. I,e spectre de fibres du tronc vagal inférieur semble devoir être très voisin.
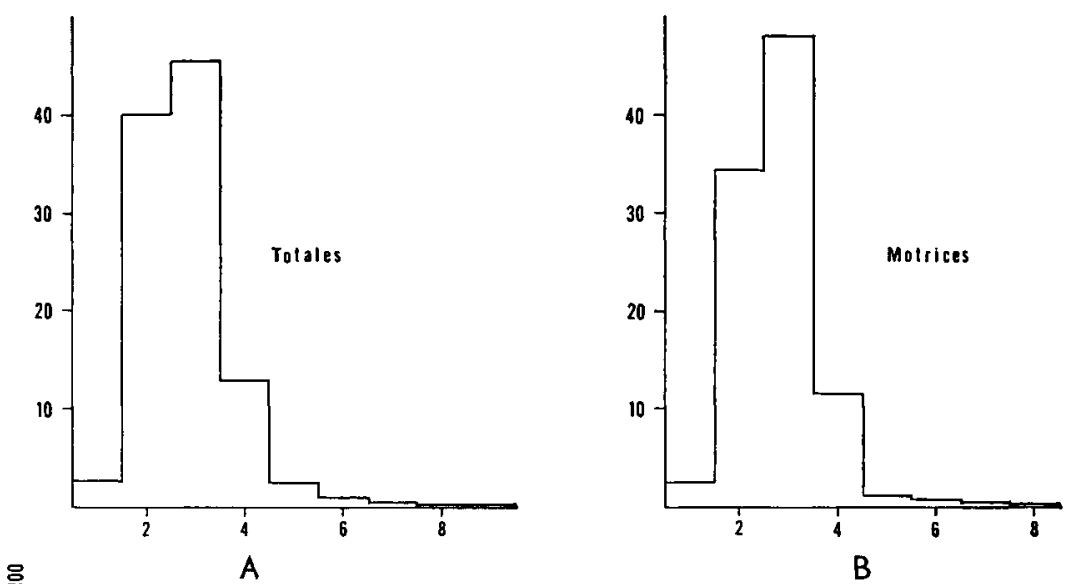

$\therefore$

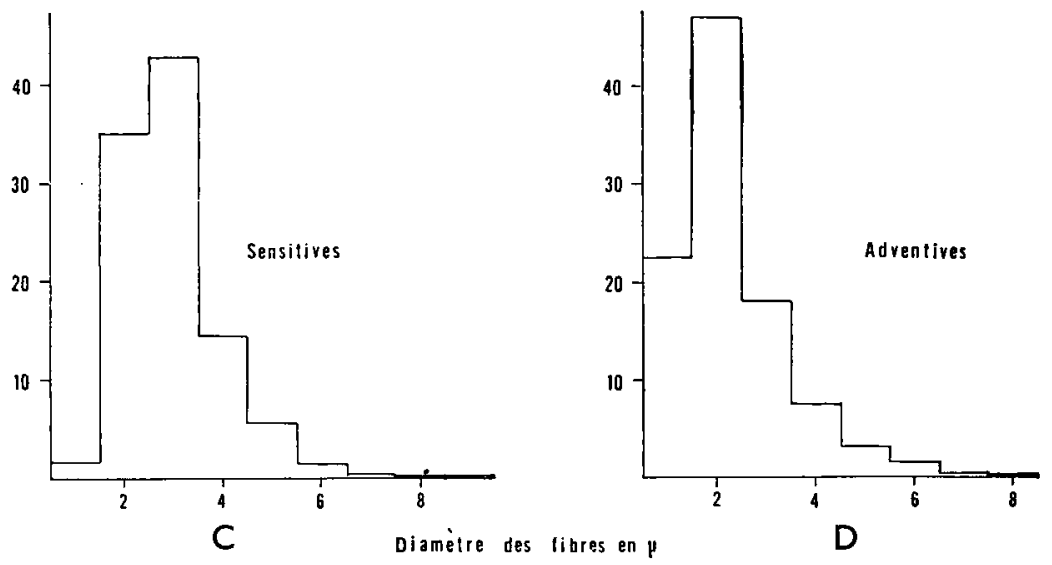

FIG. 4. - Spectre de diamètre des fibres du nerf vague thoracique supérieur.

Les divers spectres correspondent au nerf entier, aux fibres sensitives dont les neurones d'origine se trouvent dans le ganglion plexiforme, aux fibres motrices dont les neurones d'origine se trouvent dans le bulbe, enfin aux fibres adventives dont l'origine est discutée dans le texte.

Remarquer que les fibres sensitives et les fibres motrices ont un spectre très voisin. 


\section{Discussion}

Chez le Mouton, Habel (I956) a trouvé, dans le vague thoracique, Io à 30 p. Ioo de fibres amyéliniques, 60 à $70 \mathrm{p}$. Ioo de fibres myélinisées ayant un diamètre inférieur à $2 \mu$, et Io à $30 \mathrm{p}$. Ioo de fibres myélinisées ayant un diamètre compris entre 2 et $4 \mu$.

IGGO (I956) a estimé à plusieurs milliers le nombre des fibres vagales myélinisées qu'il a trouvées au niveau diaphragmatique dans le vague du Mouton ou de la Chèvre. Chez un mouton, 80 p. roo des fibres avaient de 2 à $4 \mu$ de diamètre, I4 p. Ioo avaient de 4 à $6,5 \mu$, et $3 p$. Ioo avaient de 7 à $12 \mu$.

Fn ce qui concerne les fibres myélinisées nos chiffres sont très proches de ceux de IGGo, et un peu moins de ceux de HABEL qui a vu les fibres plus fines que nous. Mais il faut noter que IGGo a utilisé, comme nous, une coloration à l'acide osmique, tandis que HABEL a fait une imprégnation argentique selon la technique de Hor,MEs (I943). Nous n'avons pas d'idée personnelle concernant le nombre de fibres amyéliniques que peut contenir le vague du Mouton, mais les connaissances de la structure des fibres amyéliniques acquises dans ces dernières années grâce au microscope électronique doivent nous inciter à considérer les comptages avec la plus extrêtne prudence. Selon GASSER (I955), le point noir que l'on obtient avec les techniques argentiques, et que l'on compte normalement pour une fibre, peut être en réalité un paquet de 2 à 5 fibres, ou plus, que le pouvoir de résolution du microscope optique ne permet pas de séparer.

Chez les animaux de laboratoire courants (Chat, Lapin, Chien) et chez 1'Homme, contrairement à ce que l'on voit chez les Ruminants, les branches vagales destinées à l'abdomen sont essentiellement composées de fibres amyéliniques. Ce fait avait été signalé depuis longtemps (Gaskel, I886; Chase et Ranson, I9I4; Raxson, I9I4b pour le Chien; HeINBECKER et O'LEARY, I933, etc.). Mais des résultats plus précis ont été fournis récemment. Ainsi chez le Lapin, il y aurait au niveau du diaphragme selon Evans et Murray (I953), 20 à 30 ooo fibres dont 60 myélinisées. Chez le Chat, selon Agostoni et al. (I957), il y aurait en moyenne $3 \mathrm{I}$ ooo fibres dont 400 myélinisées. Chez le Chat également, Mohruddix (I953) a trouvé dans un nerf droit 535 fibres myélinisées dont $93 \mathrm{p}$. Ioo avec un diamètre compris entre 2 et $3 \mu$. Enfin chez 1'Homme, Schnitzleis et al. (I958) ont trouvé en moyenne 2035 fibres myélinisées pour la totalité des branches vagales, considérées au niveau du diaphragne (extrêmes : 536 et $5 \mathrm{r} 7 \mathrm{I}$ ) ; il s'agissait essentiellement de fibres ayant un diamètre égal ou inférieur à $3 \mu$.

En dépit des derniers résultats concernant 1'Homme, on pouvait être tenté d'expliquer la myélinisation importante des fibres du vague thoracique chez le Mouton soit par la taille des animaux, soit par la physiologie particulière de la motricité des préestomacs. Mais trois ordres de faits s'opposent à ces conclusions :

$I^{\circ}$ Chez un Cheval pesant environ $45^{\circ} \mathrm{kg}$, nous avons constaté que les branches abdominales du vague, pourtant très grosses, contenaient peu de fibres myélinisées. Nous en avons compté 752 dans le vague supérieur et 245 dans le vague inférieur. Les branches anastomotiques entre ces deux nerfs n'ont pas été examinées.

$2^{\circ}$ Chez un Porc de $\mathrm{x} 20 \mathrm{~kg}$, nous avons estimé à 43,000 le nombre total des fibres myélinisées contenues dans le vague thoracique, pris en arrière du récurrent.

$3^{\circ}$ Enfin, chez le Mouton, la branche nerveuse destinée à la caillette, organe dont la 
physiologie est très semblable à celle de l'estomac des Monogastriques, est elle aussi riche en fibres myélinisées.

On peut donc conclure que les branches du pneumogastrique destinées à l'abdomen contiennent chez les moutons une proportion particulièrement élevée de fibres myélinisées, et que cette myélinisation ne semble être en rapport, ni avec la taille des animaux, ni avec les particularités de la motricité des préestomacs.

\section{3. - Le vague thoracigue après une section cervicale}

\section{Résultats}

En principe le neurone d'origine des fibres vagales se trouve soit dans le bulbe, soit dans les ganglions (jugulaire et plexiforme). Par conséquent, en réalisant une vagotomie cervicale au-dessous du ganglion plexiforme, on devrait faire dégénérer la totalité des fibres du vague périphérique. En réalité, on retrouve toujours dans le vague thoracique un certain nombre de fibres intactes, en nombre variable. Chez trois animaux nous avons trouvé dans le nerf vague supérieur gauche respectivement 73,79 et 500 de ces fibres que nous appellerons adventives. La figure $4 \mathrm{D}$ représente leur spectre de diamètre (cas du nerf qui en contenait 500). Le spectre considéré est décalé vers la gauche par rapport à celui du pneumogastrique normal.

En ce qui concerne l'origine de ces fibres, nous ne disposons actuellement que d'un seul fait qui puisse aider à l'établir : chez un animal, nous avons trouvé dans le vague, à la base du cou, un amas de cellules nerveuses typiques groupées en un petit ganglion.

\section{Discussion}

L'existence de fibres "adventives » dans le vague thoracique a déjà été signalée dans d'autres espèces. Selon Evans et Murray (I954) elles constituent 5 p. Ioo du nombre total des fibres et $50 \mathrm{p}$. Ioo des fibres myélinisées quel'on trouve dans le vague abdominal du Lapin. Pour Agostonr et al. (I957), elles constituent to p. roo du nombre total des fibres présentes dans le vague abdominal du Chat.

Evans et Murray (1954) suggèrent deux origines possibles pour ces fibres, qui pourraient être soit des fibres d'origine sympathique quittant la chaîne sympathique pour rejoindre le vague dans le thorax (MOL,HANT, I9I3), soit des fibres afférentes viscérales, quittant le tronc vagal thoracique pour rejoindre les nerfs intercostaux près des trous de conjugaison des vertèbres, et entrant dans la moelle épinière par les racines dorsales (HARPER et al., r935). AgOSTONI et al. (1957), admettent que les fibres adventives sont des fibres sympathiques.

Pour notre part nous retiendrons deux hypothèses. Ces fibres nous paraissent être, ou des fibres sympathiques issues du ganglion stellaire (МонIUdDIN, I953) et destinées au cardia (JouRDAN, 1957), ou les fibres des cellules ganglionnaires erratiques signalées dans le nerf vague au-dessous du ganglion plexiforme. Nous voudrions insister sur cette dernière hypothèse qui ne semble pas encore avoir été envisagée.

L'existence de neurones disséminés dans le tronc vagal et dans ses branches a été constatée par de nombreux auteurs. Le fait aurait déjà été observé par REMAK (1844). Parmi les travaux récents, nuus pouvons citer ceux de Dolgo-Sabtroff (I935-I937), Brown (I936), Botar et al. (I950), HoffMan et Kuntz (I957). 
Tout particulièrement intéressants sont ceux de Dolgo-SaburofF. Selon cet auteur, les cellules vagales erratiques sont de 2 types. Certaines, trouvées essentiellement au niveau cervical et dans la partie antérieure du thorax, sont des cellules unipolaires analogues à celles des ganglions jugulaire et plexiforme, et seraient probablement des cellules sensitives. D'autres sont des cellules multipolaires, analogues aux cellules du type I de Dogiel trouvées habituellement dans le plexus d'AuERBach ; on trouve à leur contact des appareils péricellulaires qui dégénèrent lorsque le nerf est séparé du bulbe. Ces cellules pourraient donc être des cellules ganglionnaires de type intramural qui ont fait une migration incomplète au cours de l'embryogenèse. Les fibres adventives trouvées dans le vague pourraient donc être des fibres post ganglionnaires.

Voici ce que nous avons trouvé dans la bibliographie au sujet des cellules aberrantes dans le vague des Ruminants. Selon Chauveau et Arloing (I87I), Lavocat aurait décrit un ganglion dans le nerf droit du rumen, mais eux-mêmes n'ont pas pu le trouver. Christ (I930) a coloré avec le błeu de méthylène des cellules ganglionnaires tout le long du vague cervical des Bovins et observa un renflement ganglionnaire à l'entrée du thorax; il nie l'existence de cellules dans les nerfs superficiels du réseau. KOHLER (I952) n'a pas pu trouver de ganglions sur le vague à l'entrée du thorax. HABEI, (I958) a vu des cellules ganglionnaires, isolées ou réunies en petits groupes, dans toutes les branches abdominales du vague qu'il a étudićes, mais il ne les a pas dénombrées; chez un mouton il a trouvé un amas d'au moins 50 cellules ganglionnaires dans le vague cervical, juste avant l'entrée de celui-ci dans le thorax. Ces cellules ressemblaient plus à des cellules ganglionnaires entériques qu'aux cellules du ganglion plexiforme du même animal.

Il n'est donc pas impossible que certaines des fibres "adventives" que nous avons observées chez le Mouton soient des fibres parasympathiques post-ganglionnaires.

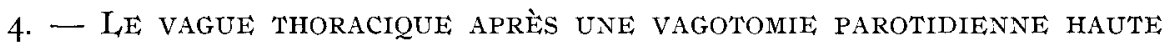

Puisque le ganglion jugulaire ne fournit pas de fibres pour le vague thoracique, en sectionnant le pneumogastrique entre le ganglion plexiforme et le crâne, nous devons faire dégénérer les seules fibres motrices. Le vague ne doit plus contenir dans ce cas que les fibres sensitives et les fibres que nous avons appelées adventives. Par comparaison avec le nerf normal, il nous est possible de connaitre le spectre de diamètre des fibres motrices disparues.

\section{A. - Absence de neurones moteurs dans le ganglion plexiforme}

\section{Résultats}

Afin de vérifier que le ganglion plexiforme n'est pas une source de fibres mctrices, nous avons sectionné le vague entre le bulbe et le ganglion plexiforme, puis, ayant laissé aux fibres issues des cellules bulbaires le temps de dégénérer, nous avons cherché si la stimulation électrique du nerf dans son trajet cervical, chez l'animal anesthésié, était susceptible de produire une contraction du réseau. Chez deux moutons, pour lesquels le temps de dégénérescence du nerf n'avait été que de six jours, nous avons obtenu une petite contraction du réseau. Chez deux autres animaux, avec un temps de dégénérescence supérieur à 15 jours, les expériences ont été négatives. 
Nous avons repris la même expérience chez un bouc, avec un temps de dégénérescence de un mois et demi, mais en réalisant une stimulation du nerf dans son trajet thoracique. De plus, l'animal était curarisé et mis en respiration artificielle. En arrêtant la pompe au moment de la stimulation, de manière à avoir une ligne de base parfaitement stable, nous avons constaté que la stimulation produisait une toute petite contraction du réseau.

\section{Discussion}

Morgan et Goland (I932) chez le Chien, Heinbecker et O'Leary (I933) et JoNEs (I937) chez le Chat, avaient admis l'existence de cellules efférentes dans le ganglion plexiforme. Ces résultats n'ont pas été retrouvés par MCSWINEY et SPURRELL, (I933) et par Agostoni et al. (I957) chez le Chat, par Evans et Murray (I954) chez le Lapin, ni par IGGo (I956 a) chez le Mouton.

En première approximation nous avons obtenu nous aussi des résultats négatifs. Ils ne peuvent pas s'expliquer par une lésion des cellules du ganglion plexiforme puisque nous avons montré que ces cellules ne manifestent aucun signe de dégénérescence après la section du vague entre le bulbe et le ganglion. D'ailleurs Downman et al. (I953), Brown et PAscoe (I954), Eccles et al. (I958), Kiraly et KRnjevic (1959), etc. ont montré que les fibres nerveuses appartenant à une cellule en voie de dégénérescence rétrograde ont une vitesse de récupération réduite, mais sont cependant capables de conduire des influx.

Cependant, nous avons observé quelquefois une toute petite réponse des estomacs. Nous n'avons pas approfondi l'étude de ce phénomène, que son infime amplitude permet de considérer comme tout à fait accessoire. Mais nous avons vu que l'existence de fibres post ganglionnaires dans le tronc vagal était possible. Il serait alors plausible de leur attribuer la toute petite contraction que nous avons obtenue.

En conclusion, nous pouvons donc considérer, avec une bonne approximation, que le ganglion plexiforme ne donne pas naissance à des fibres efférentes.

\section{B. - SPECTRE DE DiAMìtre DES FIBRES DU NERF}

\section{Résultats}

Après dégénérescence des fibres d'origine bulbaire, nous avons compté dans un vague thoracique supérieur gauche 3995 fibres myélinisées. Le nerf homologue droit normal en contenait I 2300 ; on peut supposer que ce dernier chiffre correspond également à ce qu'il y avait dans le nerf gauche avant la dégénérescence des fibres motrices. Si 1'on admet que 250 des 3995 fibres restant à gauche sont des fibres adventives, on peut calculer que les fibres myélinisées du nerf thoracique supérieur comprennent 2 p. Ioo de fibres adventives, $30 \mathrm{p}$. Ioo de fibres sensitives issues du ganglion plexiforme, et 68 p. Ioo de fibres efférentes d'origine bulbaire.

En nous servant des spectres de diamètre des fibres des trois nerfs utilisés pour le calcul ci-dessus, on peut admettre, pour la répartition des fibres de différents diamètres entre les trois catégories (adventives, motrices, sensitives), les chiffres du tableau I. On en déduit pour chacune des catégories les spectres de diamètres de la figure 4 . 


\section{TABLEAU I}

Répartition des fibres myélinisées de différents diamètres entre les trois catégories : efférentes, sensitives, adventives

Pour faire ce tableau nous avons utilisé les résultats obtenus par l'étude d'un nerf thoracique supérieur gauche dégénéré (section entre le crâne et le glanglion plexiforme) et par celle du nerf droit homologue du même mouton. Le calcul suppose que ces deux nerfs étaient primitivement identiques.

Les valeurs données pour les fibres adventives sont très approximatives, mais une erreur sur ces chiffres ne peut entrâ̂ner une conséquence que pour le nombre des fibres sensitives dont le diamètre est inférieur à $I, 5 \mu$.

\begin{tabular}{|c|c|c|c|c|c|c|c|c|c|c|c|}
\hline \multirow{3}{*}{\begin{tabular}{c}
\multicolumn{1}{c}{$\begin{array}{c}\text { Catégorie } \\
\text { des } \\
\text { fibres }\end{array}$} \\
Efférentes.........
\end{tabular}} & \multicolumn{10}{|c|}{ Nombre de fibres des différents diamètres } & \multirow{3}{*}{$\begin{array}{c}\text { Nombre total } \\
\text { de fibres } \\
\text { myélinisées }\end{array}$} \\
\hline & \multicolumn{2}{|c|}{1,5} & 2,5 & 3,5 &, 5 & 5,5 & 6,5 & ,5 & \multicolumn{2}{|c|}{$9,5: \mu$} & \\
\hline & 211 & 2869 & 4045 & 990 & 93 & 59 & 38 & 12 & 3 & 3 & \\
\hline Sensitives ....... & 62 & 1348 & 1521 & 552 & 216 & 60 & 11 & 4 & 3 & 0 & 3745 \\
\hline Adventives....... & 56 & 117 & 45 & 19 & 8 & 4 & 1 & 0 & 0 & 0 & 250 \\
\hline Total ....... & 330 & 4304 & 5608 & 1561 & 317 & 123 & 50 & 16 & 6 & 3 & 12315 \\
\hline
\end{tabular}

Nous avons estimé à $\mathrm{I} 700$ le nombre des fibres myélinisées qui persisteraient après une bivagotomie haute dans un tronc vagal inférieur, alors qu'un nerf normal en contient environ 8000 à to ooo. La proportion des fibres sensitives paraît donc plus faible dans le vague thoracique inférieur que dans le supérieur.

\section{Discussion}

a. - La première publication qui contient un comptage différentiel des fibres afférentes et efférentes du vague abdominal est celle de Evans et Murray (I954); ils ont travaillé sur le Lapin. Une étude semblable a été faite chez le Chat par AGosTONI et al. (I957). Nous avons groupé dans le tableau 2, les valeurs trouvées par ces auteurs et celles que nous pouvons envisager pour le Mouton.

\section{TABLEAU 2}

Nombre des fibres nerveuses de différents types contenues dans les vagues thoraciques au moment oit ils traversent le diaphragme

Nous avons emprunté à Evans et Murray (1954) les valeurs pour le Lapin, à Agostoni et al. celles pour le Chat. Les chiffres ont été arrondis.

\begin{tabular}{|c|c|c|c|}
\hline \multirow{2}{*}{ Types de fibres } & \multicolumn{3}{|c|}{ Nombre total de fibres au niveau du diaphragme } \\
\hline & Lapin & Chat & Mouton \\
\hline \multicolumn{4}{|l|}{ Fibres efférentes : } \\
\hline amyéliniques ... & 2600 & 3100 & $?$ \\
\hline myéliniques ....... & 0 & 0 & 22000 \\
\hline Fibres sensitives: & & & \\
\hline amyéliniques..$\ldots \ldots \ldots \ldots \ldots \ldots \ldots \ldots$ & 22000 & 25000 & $?$ \\
\hline myéliniques $\ldots \ldots \ldots \ldots \ldots \ldots \ldots \ldots \ldots$ & 30 & 360 & 9000 \\
\hline Fibres adventives: & & & \\
\hline amyéliniques . . . . . & 1200 & 3100 & $?$ \\
\hline myéliniques.......... & 30 & 40 & 500 \\
\hline
\end{tabular}


Mohruddin (I953) chez le Chat, n'a pas fait de travail quantitatif précis. Cependant il a examiné des vagues thoraciques quelques jours après la section intra-crânienne du vague, et a constaté la présence d'un certain nombre de fibres myélinisées en cours de dégénérescence. Les conclusions d'Agostoni et al. basées uniquement sur une méthode soustractive doivent donc être quelque peu atténuées : il y a probablement chez le Chat un certain nombre de fibres efférentes qui sont myélinisées (plusieurs centaines selon MOHIUDDrN). En se basant sur le nombre de cellules du ganglion plexiforme et du noyau dorsal moteur du pneumogastrique qui présentent des figures de dégénérescence rétrograde à la suite d'une vagotomie thoracique gauche, Morruddin arrive à la conclusion qu'il y aurait à gauche environ 3 ooo fibres motrices et de 4 ooo à 6 ooo fibres sensitives, proportion très différente de celle obtenue par Evans et Murray (I954) ou pat Agostoni, Chinnock, Daly et Murray (I957). Lue travail de ces derniers auteurs paraît plus précis que celui de MoHIUdDin. Mais nous avons déjà dit quelles réserves appellent les comptages de fibres amyéliniques. Les risques d'erreurs sont particulièrement graves pour l'estimation du nombre des fibres motrices, qui est faite à l'aide d'une méthode soustractive. En effet, si un faisceau nerveux non résolvable au microscope optique comprend uniquement des fibres motrices, il disparaît complètement après dégénérescence de ces fibres. Mais s'il existe, ce qui paraît probable, des faisceaux mixtes comprenant à la fois des fibres motrices et des fibres sensitives, ils subsistent après dégénérescence des fibres motrices, et la disparition de ces dernières passe inaperçue.

b. - Chez les Ruminants, personne avant nous n'avait fait une étude histologique différentielle des fibres motrices et sensitives du pneumogastrique. Nous disposons cependant d'un certain nombre de données physiologiques.

IGGo (I955) a mesuré chez la Chèvre la vitesse de conduction de quatre fibres sensitives vagales d'origine gastrique isolées $i n$ situ. Il a trouvé des vitesses de 2, 5, 6 et 12 mètres par seconde. IGGo (I956 $b$ et I958) note que dans deux fibres les vitesses de conduction étaient de 5 et 2 mètres par seconde lorsque les électrodes de stimulation étaient cervicales, et respectivement de 2 et I, 3 mètres par seconde lorsque les électrodes de stimulation étaient dans le thorax (les électrodes réceptrices étaient au cou). Pour expliquer cette différence il admet que le diamètre de ces deux fibres n'était pas uniforme sur toute leur longueur, et que l'une au moins devait être myélinisée dans son trajet cervical, et amyélinique dans le thorax.

Par l'étude du potentiel complexe provoqué par la stimulation électrique du nerf cervical, IGGo (I956 a) a estimé que les fibres motrices ont une vitesse de conduction comprise entre I et I 6 mètres par seconde. L'augmentation de l'intensité de la stimulation au-dessus du seuil des fibres amyéliniques n'augmentait pas la force de la contraction du réseau, ce qui semblait indiquer qu'il n'existe pas de fibres motrices amyéliniques. Cependant, si IGGO a bien démontré qu'aucune des fibres qui sont déjà amyéliniques au niveau cervical n'est motrice pour les estomacs, il n'a pas fourni d'arguments à l'encontre de l'hypothèse selon laquelle certaines des fibres amyéliniques trotvées par HABEL dans le vague thoracique seraient des fibres motrices myélinisées au niveau cervical.

c. - Compte tenu de ces données bibliographiques, que pouvons-nous penser de nos résultats histologiques? Ils sont en accord avec ceux de IGGo et de HABEL, mais ils diffèrent sensiblement de ceux obtenus chez le Lapin ou le Chat.

Nous trouvons environ 22 ooo fibres efférentes au lieu de 3 ooo. Ceci peut s'expli- 
quer par l'importance toute particulière de l'appareil gastrique des Ruminants. Mohiuddin a évalué à I2 ooo le nombre de cellules des deux noyaux moteurs du pneumogastrique chez le Chat. Or, d'après les comptages que nous avons effectués, ce nombre varie de 32 à 36 ooo chez le Mouton. Le noyat bulbaire contient donc assez de neurones pour justifier le nombre de fibres que nous trouvons dans le vague thoracique, bien que ce noyau fournisse certainement des fibres pour la trachée, l'œsophage et peut-être le coeur.

Nous ne trouvons que 9 ooo fibres sensitives contre 22 ou 25 ooo chez le Lapin et le Chat. Cette disparité paraît tout à fait anormale. On ne comprend pas pour quelles raisons l'innervation sensitive de l'estomac des Ruminants serait moins riche que celle de l'estomac des Monogastriques. Pour expliquer cette divergence il faut sans doute tenir compte des fibres amyéliniques. En admettant, avec HABEL qu'elles représentent $30 \mathrm{p}$. Ioo du nombre total des fibres, et avec IGGO qu'elles sont toutes sensitives, on peut estimer à $\mathrm{I} 4$ ooo le nombre de fibres sensitives amyéliniques et par conséquent à 23000 le nombre total des fibres sensitives contenues dans le vague thoracique du Mouton. Ce dernier chiffre est de même ordre de grandeur que celui trouvé chez le Chat ou le Lapin.

\title{
CONCLUSION
}

Nos expériences montrent que le spectre de diamètre des fibres sensitives myélinisées contenues dans les branches du nerf pneumogastrique destinées à l'abdomen est très voisin de celui des fibres motrices. Nous n'avons pas étudié les fibres amyéliniques, mais qu'elles soient toutes sensitives, comme l'admet IGGo (I956 a), ou que certaines soient sensitives et d'autres motrices, la conclusion sera la même : il est certainement illusoire d'espérer obtenir un tronc vagal purement moteur en recourant à des techniques sélectives de destruction ou de blocage physiologique portant sur les fibres nerveuses. Nous ne pourrons atteindre ce résultat que si nous trouvons le moyen de détruire les corps cellulaires du ganglion plexiforme sans léser les fibres motrices; c'est ce que nous essayons de faire actuellement.

\section{Reçu pour publication en novembre $\mathbf{1} 962$.}

\author{
SUMMARY \\ DISTRIBUTION OF THE NERVOUS SENSORY AND MOTOR FIBRES \\ IN THE VAGO-THORACIC TRUNK OF TIE SHEEP
}

The ganglion nodosum, the ganglion jugulare and the intra-thoracic branches of the vagus nerve have been studied in the Seep.

The technique of the retrograde degeneration has shown that the cellular bodies of the vagal sensory fibres to the abdomen are in the ganglion nodosum, and not in the jugular one. The vagal branches of the abdomen contain a great number of myelinated fibres (about 32000 at the diaphragmatic level). One can estimate that $83 \mathrm{p}$. 100 of them have a diameter inferior to $3,5 \mu$, and I 6 p. Ioo from 3,5 to $6,5 \mu$. The non-myelinated fibres have not been counted.

The comparison of normal nerves with nerves affected by a long "wallerienne " degeneration following a vagotomy operated either above the ganglion nodosum or in the midneck, shows that 
68 p. I00 of the fibres contained in a normal nerve are efferent fibres of bulbar origin, whereas $30 \mathrm{p}$. 100 of the fibres are sensory fibres coming from the ganglion nodosum. About $2 \mathrm{p}$. 100 of the thoracic fibres do not degenerate after a cervical vagotomy; so they have another origin, which is discussed in the text.

The sensory fibres and the motor ones have nearly the same diameter spectrum. Certainly it is not possible to destroy or block physiologically one of them without injuring or blocking the other ones, which would have been very useful in numerous experiments.

\section{RÉFÉRENCES BIBLIOGRAPHIQUES}

Agostoni E., Chinnock J. E., Daly M. de Burgh et Murray J. G, i957. Functional and histological studies on the vagus nerve and its branches to the heart, lungs and abdominal viscera in the cat. $J$. Physiol. (G. B.), 135, I82-205.

Andersson H. K., igoz. The nature of the lesions which hinder the development of nerve-cells and their processes. J. Physiol. (G. B.), 28, 499-513.

Appleton A. B. et Waites G. M. H., r957. A surgical approach to the cervical ganglion and related structures in the sheep. J. Physiol. (G. B.), 135, 52-57.

Bishop G. H., et Heinbecker P., 1935-1936. The afferent functions of non-myelinated or C fibers. Amer. J. Physiol., 114, I 79-193.

Bonvallet M. et SIGG B., I958. Étude électrophysiologique des afférences vagales au niveau de leur pénétration dans le bulbe. J. Physiol. (Fr.), 50, 63-74.

Botar J., Afra D., Moritz P., Schiffmann H. et Scholz M., I950. Die Nervenzellen und Ganglien des N. Vagus. Acta anal., 10, 284-314.

Brown G. L. et PAscoe J. E., 1954. The effect of degenerative section of ganglionic axons on transmission through the ganglion. J. Physiol. (G. B.), 123, 565-573.

Brown M. E., 1936. The occurence of sensory neurons below the ganglion nodosum of the vagus. $J$. comp. Neurol., 63, 421-429.

Cirase M. R. et Ranson S. W., I9I4. The structure of the roots, trunk and branches of the vagus nerve. J. comp. Neurol., 24, $3^{\mathrm{I}-60 .}$

Chauveau A. et Arloing S., i87 I. Traité d'anatomie comparée des animaux domestiques. $\mathbf{z}^{\mathrm{e}}$ édition, Baillière Paris.

Christ H., ı930. Der Nervus vagus und die Nervengeflechte der Vormägen der Wiederkäuer, speziell der Haube. Zischr. Zelliforsch., 11, 342-374.

Dolgo-SaburofF B., i 935 . Zur Lehre vom Aufbau des Vagussystems. I Über die Nervenzellen in dem Stämmen des N. Vagus. Z. Anat. Entw. Gesch., 105, 79-93.

Dolgo-SaburofF B., 1937. Zur Lehre vom Aufbau des Vagussystems. Mitteilung II. Über die pericellulären Apparate an den Nervenzellen in den Stämmen des N. Vagus. Z. Anat Entw. Gesch., 108, 637-647.

Dougherty R. W., Habel R. E., et Bond H. E., 1958. Esophageal innervation and the eructation reflex in sheep. Amer. J. vet. Res., 19, $155-128$.

Douglas W. W. et Malcolm J. L., I955. The effect of localized cooling on conduction in cat nerves. $J$. Physiol. (G. B.), 130, 53-7 г.

Downman C. B. B., Eccles J. C. et McIntyre A. K., 1953. Functional changes in chromatolysed motoneurones. J. comp. Neurol., $98,9-36$.

Du BoIs F. S. et Foley J. O., 1937. Quantitative study of the vagus nerve in the cat. II. The ratio of jugular to nodose fibers. J. comp. Neurol., 67, 69-87.

Dussardier M., ig6o. Recherches sur le contróle bulbaire de la motricité gastrique chez les Ruminants. Thèse Doctorat es Sciences, Paris.

Dussardier M., I961. Effets de la vagotomie intrathoracique partielle sur la survie et la croissance du Mouton. Ann. Biol. anim. Bioch. Biophys., 1 (2), I4I-I44.

Eccles J. C., Libet B. et Young R. R., 1958. The behaviour of chromatolysed motoneurones, studied by intracellular recording. J. Physiol. (G. B.), 143, I I-40.

Evans D. H. L. et Murray J. G.; 1953. Orientation of regenerating non medullated nerves. J. Physiol. (G. B.), 120, $52 \mathrm{P}$.

Evans D. H. L. et Murray J. G., 1954 a. Histological and functional studies on the fibre composition of the vagus nerve of the rabbit. J. Anat. Lond., 88, 320-337.

Evans D. H. L. et Murray J. G., I954 b. Regeneration of non-medullated nerve fibres. J. Anat. Lond., 88, $465-480$.

FOLEY J. O. et DU BOIS F., 1934. An experimental study of the rootlets of the vagus nerve in the cat. $J$. comp. Neurol., 60, I37-1 59 
GASKELL W. H., i 886 . On the structure, distribution and function of the nerves which innervate the visceral and vascular systems. J. Physiol. (G. B.), $\mathbf{7 ,} \mathbf{x}-80$.

Gasser H. S., 1955. Properties of dorsal roots unmedullated fibers on the two sides of the ganglion. $J$. gen. Physiol., 38, 709・728.

HABEL R. E., I956. A study of the innervation of the ruminant stomach. Cornell vet., 46, 555.628.

Harper A. A., McSwiney B. A., Suffolk S. F., 1935. Afferent fibres from the abdomen in the vagus nerves. J. Physiol. (G. B.), 85, 267-276.

Heinbecker P., et O'LeAry 'T., I933. The mammalian vagus nerve. A functional and histological study. Amer. J. Physiol., 106, 623-646.

Hinsey J. C., Krupp M. A. et Linamon W. T., 1937. Reaction of spinal ganglion cells to section of dorsal roots. J. comp. Neurol., 67, 205-214.

Hoffman H. H. et Kuntz A., 1957. Vagus nerve components. Anat. Rec., 127, 551-569.

IGgo A., 1955. Tension receptors in the stomach and the urinary bladder. J. Physiol. (G. B.), 128, 593-607.

IGGO A., I956 a. Central nervous control of gastric movements in sheep and goats. J. Physiol. (G. B.), 131, $248-256$.

IgGo A., $1956 b$. Afferent fibres from the viscera. XXe Congrès intern. Physiol. Bruxelles. Résumés des communications, p. 458 .

IGGO A., 1958. The electrophysiological identification of single nerve fibres, with particularieference to the slowest-conducting vagal afferent fibres in the cat. $J$. Physiol. (G. B.), 142, I Io-I 26.

Jourdan F., 1957. L'innervation du sphincter cardiaque et sa mise en jeu. J. Physiol. (Fr.), 49, 227.

Kimmel D. L. et Moyer E. K., 1947. Dorsal roots following anastomosis of the central stumps. J.comp. Neurol., 87, 289-3I9.

KIRALY J. K. et KRNJEviC K., I959. Some retrograde changes in function of nerves after peripheral section. Quart. J. exper. Physiol., 44, 244-257.

KoHler H., I952. Histologie und Histopathologie der wichtigsten vegetativen Ganglien unserer Haussäugetiere. Arch.exp. vet. Med., Dtsch., 26, 373-478.

Koreicha L. A. et Rappoport I. L,. 1929. Medicobiolog. J., 6, 3. (Cité d'après Nikiforov, i957.)

LAPORTE Y. et BEssou P., I959. Innervation afférente du muscle strié. Actualités neurophysiologiques, I $^{\text {re }}$ série, 189-201, Masson, Paris.

Lesbre F. X., I923. Précis d'anatomie comparée des animaux domestiques. Tome 11,785 p., Baillière, Paris.

Lesbre F. X. et Maignon F., I908. Contributions à la physiologie du pneumogastrique et de la branche interne du spinal. J. Physiol. Path. gén., 10, I ${ }^{\mathrm{er}}$ mémoire $377-39 \mathrm{I}, 2^{\mathrm{e}}$ mémoire $4 \mathrm{I} 5^{-429}$.

Lugaro E., I 896 . Sulle aiterazioni delle cellule nervose dei ganglia spinali in seguito al taglio della branca periferica o centrale del loro prolungamento. Rev. Pat. nerv. ment., 1, 457*470.

McSwiney B. A. et Spurrell W. R., I933. The gastric fibres of the vagus nerve. J. Physiol. (G. B.), 7r/, $447-458$.

MoHiuddin A., 1953. Vagal preganglionic fibres to the alimentary canal. J. comp. Neurol., 99, $289-318$.

Molhant M., I9I3. Le Névraxe, 15, 5 21-579.

Montane L. et Bourdelle E., I9 7 7. Anatomie régionale des animaux domestiques. II. Ruminants, $\mathrm{r}$ vol., 384 p., Baillière, Paris.

Morgan L. O. et Goland P.P., I932. Demonstration of the accelerator nerve and of post ganglionic parasympathetic fibers in the vago-sympathetic trunk of the dog. Amer. J. Physiol., 101, 274-28I.

NikIForov A. F., I957. The response of a sensory neuron to the severing of its dendrites (en russe). Dokl. Akad. Nauk. SSSR., 112, 760-763.

RANSON S. W., 1914. The structure of the vagus nerve of man as demonstrated by a differential axon stain. Anat. Anz., 46, 522-525.

Ranson S. W., Foley J. O. et Alpert C. D., i933. Observations on the structure of the vagus nerve. Amer. J. Anat., 53, 289-3 5 .

Sato J., 1933. Folio anat. japan, 11, 335. (Cité d'après Nikiforov, 1957.)

Schnitzlein H. N., Rowe L. C. et Hoffman H. H., 1958. The myelinated component of the vagus nerves in man. Anat. Rec., 131, 649-667. 